$$
\text { CONF }=950512-224
$$

UCRL-JC-119186

PREPRINT

\title{
Analytic Electrostatic Solution of an Axisymmetric Accelerator Gap
}

\author{
J. K. Boyd
}

This paper was prepared for submittal to the 1995 Particle Accelerator Conference and International Conference on High-Energy Accelerators Dallas, Texas

May 1-5, 1995

March 15, 1995

This is a preprint of a paper intended for publication in a journal or proceedings. Since changes may be made before publication, this preprint is made available with the understanding that it will not be cited or reproduced without the permission of the author. 


\section{DISCLAIMER}

This document was prepared as an account of work sponsored by an agency of the United States Government. Neither the United States Government nor the University of California nor any of their employees, makes any warranty, express or implied, or assumes any legal liability or responsibility for the accuracy, completeness, or usefulness of any information, apparatus, product, or process disclosed, or represents that its use would not infringe privately owned rights. Reference herein to any specific commercial product, process, or service by trade name, trademark, manufacturer, or otherwise, does not necessarily constitute or imply its endorsement, recommendation, or favoring by the United States Government or the University of California. The views and opinions of authors expressed herein do not necessarily state or reflect those of the United States Government or the University of California, and shall not be used for advertising or product endorsement purposes. 


\section{DISCLAIMER}

Portions of this document may be illegible in electronic image products. Images are produced from the best available original document. 



\title{
Analytic Electrostatic Solution of an Axisymmetric Accelerator Gap*
}

\author{
John K. Boyd \\ Lawrence Livermore National Laboratory, P.O. Box 808 \\ Livermore, California 94550, USA
}

\section{Introduction}

Numerous computer codes calculate beam dynamics of particles traversing an accelerating gap. In order to carry out these calculations the electric field of a gap must be determined $[1,2]$. The electric field is obtained from derivatives of the scalar potential, which solves Laplace's equation and satisfies the appropriate boundary conditions. An integral approach $[3,4]$ for the solution of Laplace's equation is used in this work since the objective is to determine the potential and fields without solving on a traditional spatial grid. The motivation is to quickly obtain forces for particle transport, [5] and eliminate the need to keep track of a large number of grid point fields. The problem then becomes one of how to evaluate the appropriate integral. In this work the integral solution has been converted to a finite sum of easily computed functions.

Representing the integral solution in this manner provides a readily calculable formulation and avoids a number of difficulties inherent in dealing with an integral that can be weakly convergent in some regimes, and is, in general, highly oscillatory.

\section{Formulation of the Scalar Potential Integral}

The Laplace equation to be solved in cylindrical coordinates for an accelerator gap is,

$$
\frac{1}{r} \frac{\partial}{\partial r}\left(r \frac{\partial \phi_{\text {total }}}{\partial r}\right)+\frac{\partial^{2} \phi_{\text {total }}}{\partial z^{2}}=0
$$

with the value of the potential prescribed at radius $a$. For axisymmetry in cylindrical coordinates; a Green's function is obtained by first expressing the delta function in terms of a trigonometric expansion [6]. The Green's function is then expanded in terms of the same functions used for the delta function expansion, but with an unknown radial function $g_{m}\left(r, r^{\prime}\right)$

$$
\begin{aligned}
G\left(\tilde{x}, \tilde{x}^{\prime}\right)= & \left(\frac{1}{2 \pi^{2}}\right) \sum_{m=-\infty}^{\infty} \int_{0}^{\infty} d k \\
& e^{i m\left(\theta-\theta^{\prime}\right)} \cos \left[k\left(z-z^{\prime}\right)\right] g_{m}\left(r, r^{\prime}\right)
\end{aligned}
$$

The equation solved by the radial function after specializing to axisymmetry, $m=0$, is as follows.

$$
\frac{1}{r} \frac{\partial}{\partial r}\left(r \frac{\partial g_{0}}{\partial r}\right)-k^{2} g_{0}=-\frac{4 \pi}{r} \delta\left(r-r^{\prime}\right)
$$

The homogeneous version of Eq.(3) is the equation for the

This work was performed under the auspices of the U.S. DOE by LLNL under contract no. W-7405-Eng-48.

DISTRIBUTION OF THIS DOCUMENT IS UNLMMTED modified Bessel functions. It is thus solved by a linear combination of $I_{0}(k r)$ and $K_{0}(k r)$ that satisfy the zero boundary condition at $r=a$ and the derivative discontinuity condition. Inserting the appropriate $g_{0}\left(r, r^{\prime}\right)$ into Eq.(2), a $G$ is found which satisfies the necessary properties and is specialized to a region with a fixed radial boundary and thus a Dirichlet boundary condition appropriate to an accelerator gap.

$$
\begin{aligned}
G=\left(\frac{2}{\pi}\right) \int_{0}^{\infty} d k \cos k\left(z-z^{\prime}\right) \frac{I_{0}\left(k r_{<}\right)}{I_{0}(k a)} \\
\left(I_{0}(k a) K_{0}\left(k r_{>}\right)-K_{0}(k a) I_{0}\left(k r_{>}\right)\right) .
\end{aligned}
$$

In Eq.(4) $r_{<}$is the minimum of $r, r^{\prime}, r_{>}$is the maximum of $r, r^{\prime}$, and both $I_{0}$ and $K_{0}$ are zero order modified Bessel functions.

Before the accelerator gap the wall potential is $\phi_{1}$ and after the gap the potential is $\phi_{2}$. The total potential is thus constructed as the sum of a constant and an unknown scalar function which is odd in $z$,

$$
\phi_{\text {total }}=\frac{1}{2}\left(\phi_{1}+\phi_{2}\right)+\phi
$$

where $\phi$ now solves Eq.(1). The integral solution for $\phi$ is,

$$
\phi(\hat{r}, \hat{z})=\frac{2}{\pi} \int_{0}^{\infty} d x \frac{I_{0}(x \hat{r})}{I_{0}(x)} \sin x \hat{z} \int_{0}^{\infty} d z^{\prime} \phi\left(1, z^{\prime}\right) \sin x z^{\prime}
$$

where Eq.(6) is a surface integral, $\phi\left(1, z^{\prime}\right)$ is the value of the potential on the wall, and pipe radius normalized variables are used, $x=a k, \hat{r}=r / a, \hat{z}=z / a$, and the normalized gap width is $\hat{w}=w /(2 a)$. Along the wall beyond the gap $\hat{z}>\hat{w}$ the potential is constant with a boundary condition of $\phi(1, \hat{z})=\left(\phi_{2}-\phi_{1}\right) / 2$. For complete generality the boundary condition in the gap where $\hat{z}<\hat{w}$ is written as a Fourier expansion,

$$
\phi(1, \hat{z})=\left(\phi_{2}-\phi_{1}\right) \sum_{n=1}^{\infty} A_{n} \sin \left(C_{n} \hat{z}\right)
$$

where $C_{n}=n \pi /(2 \hat{w})$. Using Eq.(7) in Eq.(6) it is found that

$$
\begin{gathered}
\phi(\hat{r}, \hat{z})=\left(\phi_{2}-\phi_{1}\right)\left(\phi_{0}+\sum \phi_{n}\right) / \pi \quad \text { where, } \\
\phi_{0}=\int_{0}^{\infty} d x \frac{I_{0}(x \hat{r})}{I_{0}(x)} \cos x \hat{w}\left(\frac{\sin x \hat{z}}{x}\right)
\end{gathered}
$$

is derived from the part of Eq.(6) baving $\hat{z}>\hat{w}$ and $\phi_{n}$ is 
from the gap where $\hat{z}<\hat{w}$,

$$
\begin{aligned}
& \phi_{n}=A_{n} \int_{0}^{\infty} d x \frac{I_{0}(x \hat{r})}{I_{0}(x)} \sin x \hat{z} \\
& {\left[\frac{\sin \left[\left(C_{n}-x\right) \hat{w}\right]}{C_{n}-x}-\frac{\sin \left[\left(C_{n}+x\right) \hat{w}\right]}{C_{n}+x}\right] }
\end{aligned}
$$

From the definition $\tilde{E}=-\nabla \phi$ the field solution requires derivatives of $\phi_{0}$ and $\phi_{n}$.

$$
\frac{d \phi_{0}}{d \hat{r}}=\int_{0}^{\infty} d x \frac{I_{1}(x \hat{r})}{I_{0}(x)} \cos x \hat{w} \sin x \hat{z}
$$

$$
\frac{d \phi_{0}}{d \hat{z}}=\int_{0}^{\infty} d x \frac{I_{0}(x \hat{r})}{I_{0}(x)} \cos x \hat{w} \cos x \hat{z}
$$

$$
\begin{aligned}
\frac{d \phi_{n}}{d \hat{r}}= & A_{n} \int_{0}^{\infty} d x \frac{I_{1}(x \hat{r})}{I_{0}(x)} x \sin x \hat{z} \\
& {\left[\frac{\sin \left[\left(C_{n}-x\right) \hat{w}\right]}{C_{n}-x}-\frac{\sin \left[\left(C_{n}+x\right) \hat{w}\right]}{C_{n}+x}\right] } \\
\frac{d \phi_{n}}{d \hat{z}}= & A_{n} \int_{0}^{\infty} d x \frac{I_{0}(x \hat{r})}{I_{0}(x)} x \cos x \hat{z} \\
& {\left[\frac{\sin \left[\left(C_{n}-x\right) \hat{w}\right]}{C_{n}-x}-\frac{\sin \left[\left(C_{n}+x\right) \hat{w}\right]}{C_{n}+x}\right] }
\end{aligned}
$$

The complete gap solution can now be written in terms of $\phi_{0}, \phi_{n}$ and their derivatives.

$$
\begin{aligned}
& \phi_{\text {total }}=\frac{\left(\phi_{1}+\phi_{2}\right)}{2}+\frac{\phi_{2}-\phi_{1}}{\pi}\left[\phi_{0}+\sum_{n=1}^{\infty} \phi_{n}\right] \\
& E_{r}=-\frac{\phi_{2}-\phi_{1}}{\pi a}\left[\frac{d \phi_{0}}{d \hat{r}}+\sum_{n=1}^{\infty} \frac{d \phi_{n}}{d \hat{r}}\right] \\
& E_{z}=-\frac{\phi_{2}-\phi_{1}}{\pi a}\left[\frac{d \phi_{0}}{d \hat{z}}+\sum_{n=1}^{\infty} \frac{d \phi_{n}}{d \hat{z}}\right]
\end{aligned}
$$

\section{Integral Approximation Technique}

The integrals needed to specify the solution in Eq.(14) are given in Eq.(8)-(13). All the required integrals in Eq.(8)-(13), have integrands consisting of a ratio of Bessel functions multiplying trigonometric functions. The method of obtaining the integrals in this work is to first approximate the ratio of Bessel functions and then integrate the resulting expressions. The form of the ratio which is useful for $\phi_{0}, \phi_{n}, d \phi_{0} / d \hat{z}$ and $d \phi_{n} / d \hat{z}$ comes from the following expression,

$$
\frac{I_{0}(x \hat{r})}{I_{0}(x)}=e^{-s x}\left[1+\left(e^{s x} \frac{I_{0}(x \hat{r})}{I_{0}(x)}-1\right)\right]
$$

where $s=1-\hat{r}$. The right side of Eq.(15) is an equivalent but more advantageous way of representing the ratio of $B$ Bssel functions, since the expression in parenthesis is a function that begins at zero and rises to an asymptotic value. Consequently, the approximation is to express the Bessel function ratio in . terms of a finite sum of exponentials raised to a negative power.

$$
\frac{I_{0}(x \hat{r})}{I_{0}(x)}=e^{-s x}\left[1+A_{s y m}^{(1)}\left(1+\sum_{l=1} \gamma_{l}^{(1)} e^{-\delta_{l}^{(1)} x}\right)\right]
$$

In like manner the form of the Bessel function ratio for $d \phi_{0} / d \hat{r}$ and $d \phi_{n} / d \hat{r}$ comes from the following expression.

$$
\frac{I_{1}(x \hat{r})}{I_{0}(x)}=e^{-s x}\left[e^{s x} \frac{I_{1}(x \hat{r})}{I_{0}(x)}\right]
$$

Again the approximation is to express the Bessel function ratio in terms of a finite sum of exponentials.

$$
\frac{I_{1}(x \hat{r})}{I_{0}(x)}=e^{-s x}\left[A_{s y m}^{(2)}\left(1+\sum_{l=1} \gamma_{l}^{(2)} e^{-\delta l^{(2)} x}\right)\right]
$$

To illustrate what has been gained by the approximation, the new form of $\phi_{0}$ is examined in detail. Substituting Eq.(16) into Eq.(8) yields,

$$
\phi_{0}=\int_{0}^{\infty} d x e^{-s x}\left[1+A_{s y m}^{(1)}\left(1+\sum_{l=1} \gamma_{l}^{(1)} e^{-\delta_{l}^{(1)} x}\right)\right] \cos x \hat{w} \frac{\sin x \hat{z}}{x}
$$

where now $\phi_{0}$ is expressed in terms of a sum of integrals. The 
fundamental integral is,

$$
I^{(1+)}(s, \hat{w}, \hat{z})=\int_{0}^{\infty} d x e^{-s x} \cos x \hat{w}\left(\frac{\sin x \hat{z}}{x}\right)
$$

which depends on the normalized gap width, radial and axial position. The important observation to make is that $\mathrm{Eq}$.(22) is the Laplace transform of $\cos x \hat{w}(\sin x \hat{z} / x)$. If an auxiliary integral having a well known Laplace transform is defined,

$$
I^{(1)}(s, \hat{z})=\int_{0}^{\infty} d x e^{-s x}\left(\frac{\sin x \hat{z}}{x}\right)=\operatorname{atan}\left(\frac{\hat{z}}{s}\right)
$$

it can be seen the parameter shifting property of the Laplace transform gives,

$$
\begin{aligned}
I^{(1+)}(s, \hat{w}, \hat{z}) & =\frac{\left(I^{(1)}(s-i \hat{w}, \hat{z})+I^{(1)}(s+i \hat{w}, \hat{z})\right)}{2} \\
& =\frac{1}{2}\left[\operatorname{atan} \frac{\hat{z}+\hat{w}}{s}+\operatorname{atan} \frac{\hat{z}-\hat{w}}{s}\right]
\end{aligned}
$$

Consequently $\phi_{0}$ can be written entirely in terms of $I^{(1+)}(s, \hat{w}, \hat{z})$.

$$
\begin{aligned}
& \phi_{0}=\left(1+A_{\text {sym }}^{(1)}\right) I^{(1+)}(s, \hat{w}, \hat{z}) \\
&+A_{\text {sym }}^{(1)} \sum_{l=1} \gamma_{l}^{(1)} I^{(1+)}\left(s+\delta_{l}^{(1)}, \hat{w}, \hat{z}\right)
\end{aligned}
$$

In an identical manner to the treatment of $\phi_{0}$, the Eq.(16) and (18) approximations are inserted into Eq.(9) to Eq.(13) to give approximations to those functions. In order to obtain these functions it is necessary to compute integrals similar to Eq.(19). As before these integrals can be derived from an auxiliary integral. In general there is a similar relation for the plus and minus superscript integrals.

$$
\begin{aligned}
& I^{(\mathrm{n}+)}=\frac{\left[I^{(n)}(s-i \hat{z}, \hat{w})+I^{(n)}(s+i \hat{z}, \hat{w})\right]}{2} \\
& I^{(\mathrm{n}-)}=\frac{\left[I^{(n)}(s-i \hat{z}, \hat{w})-I^{(n)}(s+i \hat{z}, \hat{w})\right]}{2 i}
\end{aligned}
$$

\section{Determination of constants for series expressions}

Having specified the integrals needed to calculate the functions in Eq.(9) to Eq.(13), the gap solution in Eq.(14) is known when the $A_{s y m}, \gamma_{l}$, and $\delta_{l}$ constants required by the fit functions in Eq.(16) and Eq.(18) are determined. There are actually two sets of constants that need to be determined, however each set is obtained in the same manner, so the solution procedure will only be discussed for the $A_{s y m}^{(1)}, \gamma_{l}^{(1)}$, and $\delta_{l}^{(1)}$ constants. By solving a non-linear set of equations, both the $\gamma_{l}^{(1)}$, and $\delta_{l}(1)$ constants can in principle be determined. The difficulty with obtaining this solution is that the $\delta_{l}{ }_{l}^{(1)}$ constants can be imaginary rather than strictly real. As a consequence, the fit function then becomes exponentials multiplied by sine and cosine functions, and this causes the fit function to be rippled. To avoid this problem the $\delta_{l}^{(1)}$ constants are specified to be known positive real numbers. Heuristically the large values of $\delta_{l}^{(1)}$ determine the fit near zero and smaller values tend to have an effect over an extended range. The set of equations that then has to be solved for a particular radius is,

$$
\sum_{l=1}^{N} \gamma_{i}^{(1)} e^{-\delta_{l}^{(1)} x_{i}}=\left(\frac{f\left(x_{i}, \hat{r}\right)}{f(100, \hat{r})}\right)-1
$$

which is just $N$ equations in $N$ unknowns. In Eq.(25) the asymptotic value is defined to be at $x=100$, and,

$$
f(x, \hat{r})=e^{s x} \frac{I_{0}(x \hat{r})}{I_{0}(x)}-i \cdots
$$

The solution procedure for Eq.(25) is to first solve for a standard lower, upper matrix decomposition. This result only depends on already specified $\delta_{l}^{(1)}$ constants and is only done once. It is then multiplied times the right hand side of Eq.(25) for particular $\hat{r}$ values that can vary. In principle Eq.(25) can be symbolically solved to obtain $\gamma_{l}^{(1)}(\hat{r})$, however the expressions rapidly get unwieldy for $N>4$.

\section{Conclusions}

The solution of Laplace's equation has been formulated in an integral form using a cylindrical coordinate Green's function. The integral form of the solution has been converted into a finite sum of readily calculable functions. This derived solution avoids the difficulties of the original, weakly convergent infinite integral with an oscillatory integrand. The main approximation that has been used in the derived solution is to write the finite sum in terms of Laplace integrals. In this framework the solution is formulated for an arbitrary variation of the electric field in the gap.

\section{References}

[1] P. Grivet, Electron Optics, (Pergamon Press, Oxford, 1972) p.164

[2] C.L. Chang et. al., Proc. Conf. Computer Codes and Linear Accelerators, (LANL, LA-11857-C, January, 1990) p.27

[3] C.K.Allen, S.K. Guhary, and M.Reiser, Proc. Computational Accelerator Physics, (AIP Proc 297, Los Alamos, NM, 1993) p. 568

[4] A.B. El-Kareh, J.C. El-Kareh, Electron Beams, Lenses and Optics, Vol I, (Academic Press, New York, 1970) p. 28

[5] J.K.Boyd, et. al.,Nuc. Instr. Meth. Phy. A272(1988)p.590

[6] J.D. Jackson, Classical Electrodynamics, (John Wiley, New York, 1975) p.118 